\title{
Intermittent rectal motor activity: a rectal motor complex?
}

\author{
A Prior, U J Fearn, N W Read
}

\begin{abstract}
Prolonged nocturnal recordings from multiple sites in the anorectum and duodenum were performed in 12 healthy volunteers to investigate the presence and determine the characteristics of nocturnal rectal motor activity and assess any synchronicity with phase III of the small intestinal migrating motor complex. Runs of phasic contractions of two contractile frequencies ( 3 or $6 /$ minute), sustained for more than three minutes, and preceded and followed by motor quiescence were observed in 10 of the 12 subjects. This phenomenon is similar to that described by other investigators and termed the 'rectal motor complex'. The runs of contractions showed considerable inter- and intrasubject variation, with a duration of 3-30 minutes (median 9.0), amplitude of 10-55 $\mathrm{mm} \mathbf{H g}$ (median 20.0), and periods of $10-420$ minutes (median 55.5) between contractile activity. There was no propagation through the rectum, the phasic motor activity rarely occurred simultaneously at more than one rectal recording site. No consistent relation with phase III of the small intestinal migrating motor complex was observed in any subject. In view of these findings, we question whether this intermittent motor activity merits the term 'rectal motor complex'.
\end{abstract}

The normal pattern of rectosigmoid motor activity remains poorly understood. Studies of this region have largely been confined to the measurement of response to stimuli and have been limited to short observation periods.' Two recent reports ${ }^{23}$ have suggested that recurrent bursts of motor activity occur in the rectum, particularly at night and during fasting. This activity has been compared with phase III of the small intestinal migrating motor complex and termed the rectal motor complex. As these reports used only single rectal recording sites, however, little is known of the propagation characteristics of rectal motor activity.

This study aimed to record contractile activity from multiple sites within the anorectum and duodenum in order to define further the characteristics of the rectal motor complex and examine its relation with the small intestinal migrating motor complex. General Hospital, Sheffield

A Prior

U J Fearn

N W Read

Correspondence to:

Dr A Prior, Department of

Gr A Prior, Department

Norwich Hospital, Bowthorpe

Road, Norwich NR2 3TU.

Road, Norwich NR2 3TU.

14 December 1990

\section{Methods}

Nocturnal rectal motor activity was recorded in 12 healthy volunteers ( 10 men and two women, age range $20-28$ years). In eight of the volunteers, small intestinal motor activity was also recorded. Subjects attended the department at $\mathbf{9 . 3 0}$ am after an overnight fast and were intubated nasally with a small intestinal catheter that had two recording sites separated by $15 \mathrm{~cm}$. Using the transmucosal potential difference to assess the position of the pylorus, ${ }^{+}$the catheter was guided into the duodenum and was secured to the subject's cheek when the proximal port was 15 $\mathrm{cm}$ distal to the pylorus. The subjects were then allowed to eat lunch and a light meal at $6 \mathrm{pm}$ and were instructed to return to the department at 10 pm. Without bowel preparation, a multilumen polyvinyl catheter with side holes at $1 \cdot 5,2 \cdot 5,4 \cdot 0$, $8 \cdot 0$, and $14 \mathrm{~cm}$ from the anal verge was placed in the rectum. Both the small intestinal and rectal catheters were perfused with water at $0.2 \mathrm{ml} /$ minute (Arndorfer Medical Specialities Inc, WN, USA) and connected by water filled transducers to a pen recorder (Ormed Ltd, Welwyn Garden City, UK). Subjects were allowed to fall asleep in a semidarkened room and motor activity was recorded continuously from both the rectum and small intestine between $10.30 \mathrm{pm}$ and $7.30 \mathrm{am}$. The conscious state of the subject was monitored throughout the night.

\section{Analysis}

All records were analysed visually. Rectal contractions were defined as a pressure rise exceeding $5 \mathrm{~mm} \mathrm{Hg}$ and lasting for longer than four seconds. Intermittent phasic rectal activity was identified when a run of regular contractions with a frequency of $>2 /$ minute was sustained for more than three minutes and preceded and followed by a period of quiescence of at least five minutes' duration. Phase III of the small intestinal migrating motor complexes was defined as the presence of at least three minutes of uninterrupted phasic pressures at a regular frequency of 10-12 per minute that occurred sequentially in the two recording sites. ${ }^{5}$ Movement artifacts were easily recognised as rapid fluctuations occurring as simultaneous events arising in all channels and were excluded from analysis.

\section{Results}

The small intestinal and rectal catheters were well tolerated by the subjects and a total of 91 hours of nocturnal sleep records were obtained (range 5-9 hours/subject, median $7 \cdot 8$ hours).

The following types of contractile activity were observed in the rectum.
RUNS OF REGULAR RECTAL CONTRACTIONS

These were observed in 10 of the 12 subjects but showed considerable intra- and intersubject variation (Figs 1-3). Their duration ranged from 3 to 30 minutes (median 9.0) and they occurred 
$14 \mathrm{~cm}$

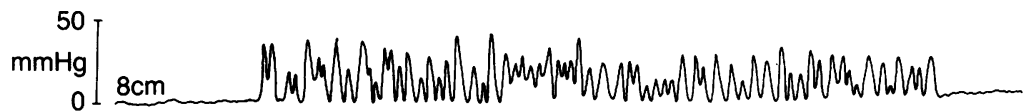

$4 \mathrm{~cm}$

$1 \mathrm{~min}$

Figure 1: Recording of intraluminal rectal pressure at three sites in the rectum showing a burst of phasic activity at $8 \mathrm{~cm}$ with quiescence at $4 \mathrm{~cm}$ and $14 \mathrm{~cm}$.

at intervals of $10-420$ minutes (median $55 \cdot 5$ ). The amplitude of the contractions varied from 10-55 mm Hg (median 20) and they occurred at two contractile frequencies (2-3/minute and 6/ minute). The characteristics of the recurrent rectal activity varied significantly depending on distance from the anal verge (Table). A total of 88 periods of phasic activity were observed; on only eight occasions was activity simultaneously present at two adjacent recording sites (Fig 3), and was then often of a different contractile frequency and duration. No evidence of propagation was seen (Fig 3 ) in any subject. No association was observed between phasic rectal contractile activity and any pattern of anal motor activity.

\section{CONTINUOUS IRREGULAR RECTAL MOTOR}

ACTIVITY

Continuous irregular rectal motor activity was observed in two subjects, while asleep, and lasted for five hours and 30 minutes respectively (Fig 3). This consisted of contractile activity of variable amplitude and frequency in which occasional short periods (less that two minutes) of quiescence occurred. In both subjects the irregular activity was observed at $8 \mathrm{~cm}$ from the anal verge and was associated with quiescence at the other two sites.

\section{SINGLE CONTRACTIONS}

Single contractions that occurred simultaneously in two or more adjacent rectal channels were observed in six of the 12 subjects. These lasted for 10-23 seconds with a frequency of $2-6 / 8$ hours and were associated with an anal relaxation on $50 \%$ of occasions.

SMALL INTESTINAL MOTOR ACTIVITY

A total of 41 small intestinal migrating motor

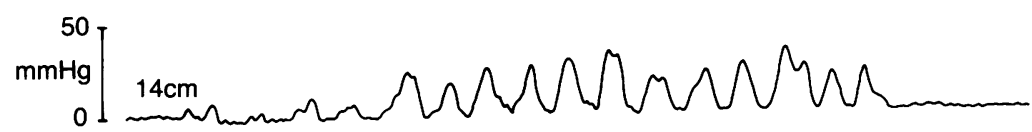

Figure 2: Recording of intraluminal rectal pressure at $14 \mathrm{~cm}$ showing a run of contractions with a frequency of 2-3/minute.
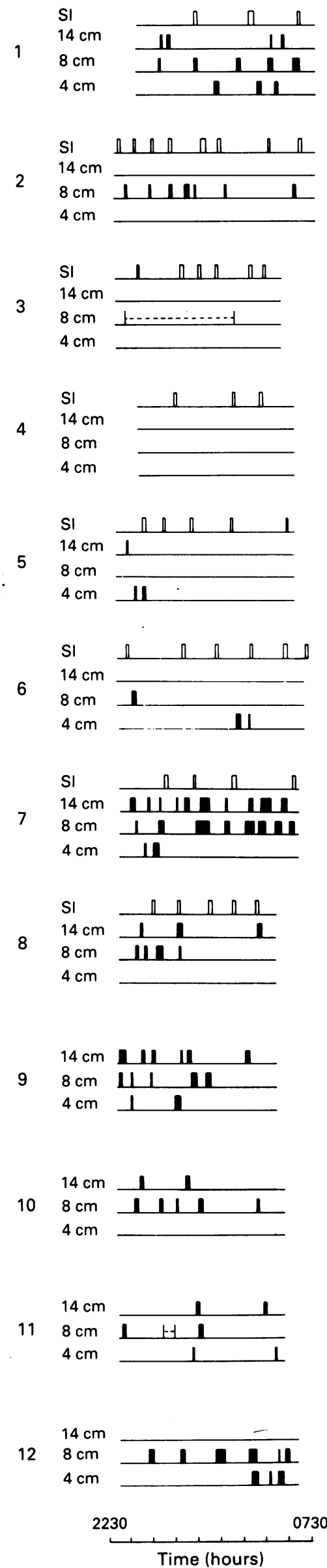

Figure 3: Distribution of rectal and small intestinal motorlactivity during sleep in all subjects. 0 small intestinal MMC; runs of rectal contractions; 1 - - - irregular rectal contractile/activity. 
Characteristics of recurrent rectal activity at three rectal recording sites

\begin{tabular}{llll}
\hline $\begin{array}{l}\text { Distance from } \\
\text { anal verge }(\mathrm{cm})\end{array}$ & $\begin{array}{l}\text { Total } \\
\text { no }\end{array}$ & $\begin{array}{l}\text { No at } \\
3 / \text { minute }\end{array}$ & $\begin{array}{l}\text { No at } \\
\text { 6/minute }\end{array}$ \\
\hline 4 & 17 & 4 & 13 \\
8 & 44 & 11 & 33 \\
14 & 27 & 24 & 3 \\
\hline
\end{tabular}

$x^{2}(\operatorname{df} 2)=31 \cdot 37 p<0 \cdot 001$

complexes were recorded from eight of the 12 subjects with a median duration of 6 minutes (range 4-15) and a cycle length that ranged from 30 to 150 minutes (median 68). No relation was observed between phase III of the small intestinal migrating motor complex and the occurrence of runs of rectal contractions or irregular motor activity (Fig 3).

\section{Discussion}

This study confirms that recurrent bursts of phasic motor activity can be identified in the human rectum during sleep. These were not evident in all subjects, however, and exhibited considerable inter- and intrasubject variation in terms of both the duration and interval between contractile activity.

Two reports on rectal motor activity have recently been published. Using an ambulatory system with a single strain gauge transducer within the rectum, Kumar et $a l^{2}$ described the occurrence of recurrent phasic activity during fasting and at night in all subjects studied and termed this the 'rectal motor complex'. Orkin et $a l,{ }^{3}$ using a water perfused catheter with a single rectal port, observed similar contractile activity in 11 of 12 subjects studied. This occurred after meals, as well as during fasting and at night, and showed no synchronicity with phase III of the small intestinal migrating motor complex or with the non-rapid eye movement (REM) sleep cycle. This study has extended these observations by showing that recurrent rectal motor activity is segmental rather than propagated, and has confirmed that there seems to be no association with the migrating motor complex cycle. In addition, recurrent rectal motor activity was noted to occur at two contractile frequencies (3 or $6 /$ minute) whereas previous reports have observed contractions at only $3 /$ minute. This difference may partly relate to the site at which rectal activity was recorded, as in our study the occurrence of contractile activity at each frequency varied according to the distance from the anal verge.

The origin of recurrent rectal activity remains unclear. Kumar $e t a l^{2}$ considered that the runs of rectal contractions represent periodic activity similar to phase III of the small intestinal migrating motor complex. As this activity seemed more noticeable during sleep, they suggested that it was a function of intrinsic rather than extrinsic innervation and concluded that monitoring of rectal motor activity may be of value in the assessment of intrinsic neural integrity in patients with anorectal dysfunction. Our observations and those of Orkin et $\mathrm{al}^{3}$ raise doubts as to the validity of these conclusions. Recurrent rectal motor activity is not nearly so characteristic a phenomenon as phase III of the small intestinal migrating motor complex, which has a well defined periodicity, contractile frequency, and migration velocity. ${ }^{5}$ In contrast, recurrent rectal contractile activity is extremely variable, with periods between adjacent contractions of as little as 10 minutes and as great as six hours. Activity is segmental rather than propagative, and may only be apparent at a single site. In this respect, the runs of rectal contractions are similar to those observed by other workers in the human transverse, descending, and sigmoid colon ${ }^{6}$ and do not resemble the cyclical migrating motor activity recorded in the dog colon. ${ }^{78}$ In addition, unlike the small intestinal migrating motor complex, rectal phasic activity is not consistently disrupted by activity in the extrinsic nerves induced by eating. These observations do not support the contention that recurrent rectal motor activity is the manifestation of an intrinsic neural rhythm. Even if this were so, the variability in occurence and characteristics in normal subjects suggests that monitoring of rectal activity is unlikely to be of value in the assessment of the intrinsic nervous system of patients with anorectal dysfunction.

The function, if any, of the runs of rectal contractions is also unknown. Orkin $e t a l^{3}$ have suggested that the phasic activity may act to keep the rectum empty, thereby avoiding the sensation of rectal fullness and the desire to defecate. How activity occurring intermittently and relatively infrequently at one site in the rectum could perform such a function is not clear.

Recurrent phasic activity was not the only phenomenon seen in the nocturnal records. Single rectal contractions occurring simultaneously at all rectal recording sites and associated with an anal relaxation were observed in $50 \%$ of the subjects. This pattern of activity resembles the rectal response to balloon distension, and may therefore be secondary to the arrival of a sudden distending stimulus such as flatus in the rectum. No high amplitude propagated contractions were observed, ${ }^{6}$ perhaps because the recordings were carried out at night when these phenomena are rarely seen. ${ }^{6}$

In conclusion, this study has confirmed that recurrent bursts of rectal motor activity do occur in the human rectum, but are segmental and show no clear evidence of cyclicity. Should so variable a phenomenon be formalised by the term 'rectal motor complex'? Does the variable gap between complexes merit the term 'periodic motor activity'? These are semantic issues, but become important if changes in this motor activity are regarded as indices of the integrity of the intrinsic nervous system. Further work is required in order to clarify the nature and control mechanisms underlying phasic rectal activity.

1 Dinoso VP, Murthy SNS, Goldstein J, Rossner B. Basal moto activity of the distal colon: a reappraisal. Gastroenterology 1983; 85: 637-42.

2 Kumar D, Williams NS, Waldron D, Wingate DL. Prolonged manometric recording of anorectal activity in ambulant human subjects: evidence of periodic activity. Gut 1989; 30: 1007-11. 3 Orkin BA, Hanson RB, Kelly KA. The rectal motor complex. Gastrointest Motility 1989; 1: 5-8.

4 Houghton LA, Read NW, Heddle R, Maddern GJ, Downton J, Toouli J, et al. Motor activity of the gastric antrum, pylorus and duodenum under fasted conditions and after a liquid meal Gastroenterology 1988: 94: 1276-84. 
5 Kellow JE, Borody TJ, Phillips SF, Tucker RL, Haddad AC. Human interdigestive motility: variations in patterns from esophagus to colon. Gastroentrology 1986; 91: 386-95.

6 Narducci F, Bassotti G, Gaburri M, Morelli A. Twenty four hour manometric recordings of colonic motor activity in healthy man. Gut 1987; 28: 17-25.
7 Schuurkes JAJ, Tukker JJ. The interdigestive colonic motor complex of the dog. Arch Int Pharmacodyn Ther 1980; 247: 329-34.

8 Sarna SK, Condon R, Cowles V. Colonic migrating and nonmigrating motor complexes in dogs. Am $\mathcal{F}$ Physiol 1984; 246 : G355-60. 